\title{
Investigation into the reproducibility of the association of cord blood magnesium concentration and cerebral palsy or death in children
}

\section{Christopher Luke Dixon}

University of Texas Medical Branch School of Medicine

\section{Xiaoxi Liu}

University of Utah

\section{Alfred Balch}

University of Utah

\section{Maged Costantine}

Ohio State University College of Medicine

Catherine M. Sherwin ( $\nabla$ catherine.sherwin@hsc.utah.edu )

https://orcid.org/0000-0002-0844-3207

\section{Research article}

Keywords: fetal neuroprotection, cerebral palsy, magnesium sulfate, cord blood, magnesium concentrations, neonatal death, death in children

Posted Date: March 27th, 2020

DOI: https://doi.org/10.21203/rs.3.rs-19283/v1

License: (c) (i) This work is licensed under a Creative Commons Attribution 4.0 International License.

Read Full License 


\section{Abstract}

Objective: To evaluate the association of cord blood magnesium concentrations at the time of birth with cerebral palsy (CP) and neonatal death.

Study Design: A secondary analysis of a randomized controlled trial that randomized women at imminent risk of delivery between 24 and 31 weeks of gestation to receive magnesium sulfate or placebo. This 'study's primary outcome was a composite of either moderate to severe CP or death. Secondary outcomes included $\mathrm{CP}$, moderate to severe $\mathrm{CP}$, neonatal death, and neonatal head ultrasound findings. We used a logistic regression model to evaluate the relationship between the concentration of magnesium in cord blood and study outcomes.

Results: A total of 668 women were included in this analysis and were randomized to magnesium sulfate at $28 \pm 2.5$ 'weeks' gestation. Cord blood magnesium concentrations were not associated with the primary outcome of infant death by 1 year of age or moderate or severe cerebral palsy, as assessed at or beyond 2 years of age (aOR $0.95(0.67,1.36), p=0.79)$. Cord blood magnesium concentrations were not associated with any of the secondary outcome measurements.

Conclusion: Cord blood magnesium concentrations were not associated with moderate to severe cerebral palsy or death, or other neurodevelopmental or sonographic outcomes.

\section{Introduction}

Cerebral palsy (CP) is a group of permanent disorders of the development of movement and posture attributed to non-progressive disturbances, which have occurred in the developing fetal or infant brain. These motor disorders are often accompanied by epilepsy and changes in sensation, perception, cognition, and behavior [1]. Recently, Maenner et al. [2] analyzed the results of two U.S. representative surveys and found the prevalence of $\mathrm{CP}$ to be in the range of 2.6 to 2.9 per 1,000 children. Those born before 28 weeks of gestation have a significantly higher prevalence ranging from 40 to 100 per 1,000 live births [1].

In the past 30 years, several studies were conducted to evaluate the role of magnesium sulfate therapy for fetal neuroprotection among women at risk of preterm birth [3-6], with the two most extensive randomized controlled trials (RCTs) showing significant reduction in moderate to severe cerebral palsy among surviving children who were exposed to magnesium sulfate in-utero $[4,6]$. Subsequently, multiple meta-analyses found a significant decrease in the risk of cerebral palsy with the antenatal use of magnesium sulfate $[3,7,8]$. In 2010, the American College of Obstetricians and Gynecologists (ACOG) and the Society for Maternal-Fetal Medicine (SMFM) published a statement "that magnesium sulfate given before anticipated early preterm birth reduces the risk of cerebral palsy in surviving infants." For those electing to use magnesium sulfate, ACOG, and SMFM recommended developing specific guidelines for use in accordance with one of the more sizeable trials [9]. 
While physicians and hospitals created protocols and treatment guidelines for the use of magnesium sulfate for fetal neuroprotection, controversies remain. Experts argue that none of the large RCTs found any difference in the rate of the primary outcome (which in many cases was a composite of death or CP) $[10,11]$. There was controversy regarding the optimal dose and timing of treatment. Trials included a range of dosing schedules with loading doses ranging from 4-6 $\mathrm{g}$ of intravenous magnesium sulfate and maintenance dosing ranging from nothing to $2 \mathrm{~g} / \mathrm{hr}$ [8]. There is not enough evidence to recommend an optimum dosing scheme, and the Cochrane meta-analysis did not find one dosing regimen superior to another $[9,12,13]$. Moreover, a sequential trial analysis and a 6-year follow up of the Australasian Collaborative Trial of Magnesium Sulphate $\left(\mathrm{ACTOMgSO}{ }_{4}\right)$ trial do not support the use of magnesium sulfate for neuroprotection $[14,15]$.

With these controversies, we sought to determine if the fetal neuroprotection of antenatal magnesium sulfate may be demonstrated by investigating the association between cord blood magnesium concentrations at the time of birth and cerebral palsy. Within the obstetric community, there is a lack of consensus related to magnesium sulfate infusion at the time of delivery or magnesium cord blood concentrations. So, this study aimed to undertake secondary data analysis to investigate the reproducibility of the association between women exposed to magnesium sulfate in utero and the lack of association with $\mathrm{CP}$ or death in infants.

\section{Methods}

This is a secondary analysis of data from the Eunice Kennedy Shriver National Institutes of Child Health and Human Development Maternal-Fetal Medicine Units (NICHD-MFMU) Network multicenter randomized trial (Beneficial Effects of Antenatal Magnesium [BEAM]). This analysis was exempt from review by the Institutional Review Board, as it met the criteria of research involving the study of existing data.

Women at imminent risk of delivery before 32 weeks of gestation were assigned to receive magnesium sulfate or placebo [6]. A total of 2,241 women were recruited from December 1997 to May 2004 at 20 clinical centers in the U.S. They were randomized to receive magnesium sulfate or placebo when they were in between their 24th and 31st completed weeks of pregnancy. The recruitment and study protocol were presented in detail in the original publication of the randomized trial [6]. Briefly, women were eligible for inclusion if they were carrying singletons or twins and were at elevated risk for preterm delivery because of rupture of membranes, advanced preterm dilation $(4$ to $8 \mathrm{~cm})$, or a planned preterm delivery [6]. Magnesium sulfate was given intravenously as a $6 \mathrm{~g}$ loading dose (over 20 to 30 minutes) followed by a $2 \mathrm{~g}$ per hour maintenance infusion and stopped after 12 hours if delivery was no longer imminent. The maintenance infusion ( $2 \mathrm{~g}$ per hour) was restarted when delivery was determined to be imminent again, and if it had been at least 6 hours, another loading dose $(6 \mathrm{~g})$ was given [6].

Women enrolled in the primary trial, who received magnesium sulfate within 6 hours of delivery, were included in the current analysis if cord blood magnesium concentration was available. Our primary outcome was defined as a composite of infant death by 1 year of age or moderate or severe cerebral 
palsy, as assessed at or beyond 2 years of age [6]. Cerebral palsy was diagnosed by a certified pediatrician or pediatric neurologist, blinded to the maternal assignment. The Gross Motor Function Classification System was used to assess the severity of cerebral palsy. Details of the diagnostic criteria for cerebral palsy used in the parent study can be found in the initial publication [6]. Secondary outcomes included any cerebral palsy at two years of age, moderate to severe cerebral palsy, neonatal death, and neonatal neurosonographic abnormalities defined as either intraventricular hemorrhage, severe intraventricular hemorrhage (grade 3 and 4), or periventricular leukomalacia.

Statistical analyses were performed using SAS Enterprise Guide 9.4 (SAS Institute Inc., Cary, NC). Descriptive demographic and baseline characteristics of the study population were reported. To explore the association between cord blood magnesium concentration and neonatal/child outcomes, logistic regression analyses were performed. The addition of covariates followed a stepwise pattern guided by the reduction of cost function $>3.83$. Clustering was adjusted for twin gestation. Convergence was reached in all model runs. Gestational age at delivery and infant gender were included in the final model. Missing data were excluded from analyses. Significance was defined as two-sided $\mathrm{P}<0.05$.

\section{Results}

Out of 2,241 women in the original study, $668(30 \%)$ received magnesium sulfate within six hours of delivery. Table 1 presents the demographic and obstetric characteristics of the study population. The mean $( \pm$ SDD) maternal age and gestational age at randomization were $26( \pm 5.8)$ years old and $28( \pm$ $2.5)$ weeks of gestation, respectively. A total of 261 (39\%) women were nulliparous, and 64 (10\%) had twin gestations. 
Table 1

Maternal characteristics of the study population

\begin{tabular}{|ll|}
\hline Characteristics & Population $(\mathbf{n}=668)$ \\
\hline Twin gestation & $64(10)$ \\
\hline Married & $328(49)$ \\
\hline Race/ ethnicity & $296(44)$ \\
African American & $243(36)$ \\
Hiscasian & $111(17)$ \\
Asian & $7(1)$ \\
Native American/Other & $11(2)$ \\
\hline Nulliparous & $261(39)$ \\
\hline Previous preterm delivery & $174(26)$ \\
\hline No prenatal care & $39(6)$ \\
\hline Smoking during pregnancy & $188(28)$ \\
\hline Alcohol use during pregnancy & $55(8)$ \\
\hline Illicit substance use during pregnancy & $70(11)$ \\
\hline Previous preterm delivery & $160(24)$ \\
\hline Age (years) & $26 \pm 5.8$ \\
\hline Pre-pregnancy BMl (kg/m $\left.{ }^{2}\right)$ & $26 \pm 6.7$ \\
\hline Gestational age at randomization (weeks) & $28 \pm 2.5$ \\
\hline Education level (years) & $12 \pm 2.5$ \\
\hline Cervical dilation (cm) & $1.6 \pm 1.9$ \\
\hline Time since rupture (hours) & $101.6(28.9-242.1)$ \\
\hline BMl = body mass index & \\
\hline Data are reported as mean \pm standard deviation, median (interquartile range) or $\mathrm{n}(\%)$. \\
\hline
\end{tabular}

The mean time from magnesium dose initiation until birth was $0.13 \pm 0.60$ hours (Table 2). The mean \pm SD cord blood magnesium concentration was $2.98 \pm 0.81 \mathrm{meq} / \mathrm{L}$ (Table 2). While studies regarding reference ranges for cord blood magnesium concentrations in preterm infants are limited, the normal reference range reported is from 1.34 to $1.92 \mathrm{meq} / \mathrm{L}$. [16] Table 3 represents the association of cord blood magnesium concentration and the outcomes. Gestational age at delivery was associated with the primary outcome of infant death by 1 year of age or moderate or severe cerebral palsy, as assessed at or beyond 2 years of age, and various secondary outcomes. With every week increase in gestational age, the 
risk of primary outcome decreased by $6 \%(\mathrm{OR} 0.94,95 \% \mathrm{Cl} 0.92-0.96 ; \mathrm{p}<0.001)$. Additionally, female gender was associated with lower odds of developing the primary outcome (OR 0.59, 95\% $\mathrm{Cl} 0.36-0.96$; $p=0.03)$. However, cord blood magnesium concentration was not associated with the primary outcome (OR $0.95(0.67,1.36), p=0.79)$, or any other secondary outcomes.

Table 2

Descriptive statistics of magnesium sulfate concentration and administration

\begin{tabular}{|lc|}
\hline & Mean \pm SD \\
\hline Cord blood $\mathrm{Mg}(\mathrm{meq} / \mathrm{L})$ & $2.98 \pm 0.81$ \\
\hline Total drug given $(\mathrm{g})$ & $39.3 \pm 23.0$ \\
\hline Time from dosing to birth $(\mathrm{hr})$ & $0.13 \pm 0.60$ \\
\hline $\mathrm{Mg}=$ magnesium; SD = standard deviation \\
\hline
\end{tabular}

Table 3

Association between cord blood magnesium level and neonatal/child outcomes

\begin{tabular}{|llc|}
\hline & $\begin{array}{l}\text { Adjusted Odds Ratio } \\
(95 \% \mathrm{Cl}) \#\end{array}$ & P value \\
\hline Primary composite outcome* & $0.95(0.67,1.36)$ & 0.79 \\
\hline Neonatal death & $0.92(0.62,1.37)$ & 0.67 \\
\hline Cerebral palsy & $1.30(0.69,2.44)$ & 0.42 \\
\hline Moderate to severe cerebral palsy & $1.10(0.53,2.26)$ & 0.80 \\
\hline Intraventricular hemorrhage & $0.86(0.65,1.15)$ & 0.31 \\
\hline Severe intraventricular hemorrhage & $1.36(0.57,3.25)$ & 0.49 \\
\hline Periventricular leukomalacia & $0.58(0.31,1.10)$ & 0.10 \\
\hline * A composite of infant death by 1 year of age or moderate or severe cerebral palsy, as assessed at or \\
beyond 2 years of age.
\end{tabular}

\section{Discussion}

Our secondary analysis of the NICHD-MFMU network BEAM trial showed that cord blood magnesium concentrations were not associated with moderate to severe cerebral palsy or neonatal death, or other neurodevelopmental or neurosonographic outcomes. These results, along with the differing magnesium 
dosing regimens in the fetal neuroprotection trials, emphasize the need for additional research to discover the ideal dosing scheme.

The majority of the controversy regarding the use of antenatal magnesium sulfate for the prevention of cerebral palsy revolves around the lack of a significant difference in the primary outcomes of the three largest RCTs and the different dosing schemes used in these, and other trials [4-6]. As stated in a 2009 clinical opinion, "the NICHD MFMU network trial took ten years and cost \$25 million. It is, therefore, unlikely that another, larger trial will ever be conducted [17]." If this is the case, it is increasingly important to address the magnesium sulfate dosing regimen through the analysis of existing data. ACOG and SMFM recommend developing specific guidelines for use in accordance with one of the more extensive trials [9]. Identifying an optimal dosing scheme will help to clear up these vague recommendations.

Brookfield et al. [18] published a prospective pharmacokinetic study evaluating the profile of magnesium sulfate in preeclamptic and non-preeclamptic women. Using the dosing regimen of a $4 \mathrm{~g}$ loading dose and $2 \mathrm{~g} /$ hour maintenance dose, the steady-state concentration of magnesium sulfate was $7.2 \mathrm{mg} / \mathrm{dL}$ in preeclamptic women compared with $5.1 \mathrm{mg} / \mathrm{dL}$ in non-preeclamptic women. The mean gestational age of the women enrolled was 33.7 weeks of gestation, and $83 \%$ of them were preeclamptic. Using this pharmacokinetic model, Brookfield et al. [19] applied it to the BEAM cohort. They concluded that a maternal serum magnesium concentration between 3.7 and $4.4 \mathrm{mg} / \mathrm{dL}$ at delivery was associated with the lowest probability of delivering an infant with cerebral palsy. It was also noted that the total administered magnesium dose associated with the lowest probability of an infant developing cerebral palsy was $64 \mathrm{~g} \mathrm{(95 \%} \mathrm{Cl} 30$ to 98). Methodological limitations were due to interruptions in dosing and access to only the pre-pregnancy body mass index for covariate analysis. Limitations not discussed consist of the decision to include women in both treatment and placebo arms in the analysis, and why a maternal magnesium concentration above $4.4 \mathrm{mg} / \mathrm{dL}$ would confer a higher risk of delivering an infant with cerebral palsy. The pharmacokinetics of umbilical cord magnesium sulfate concentrations were also not investigated.

Initially, there were concerns regarding the safety of magnesium sulfate due to earlier findings of increased neonatal mortality [20]. Subsequent studies and meta-analyses found this to be a false association [3-8]. Our analysis confirms the lack of association between cord blood magnesium concentrations and neonatal death. Additionally, maternal safety has been extensively studied, and there is no significant difference in maternal deaths or major maternal adverse events between those exposed to magnesium sulfate or not $[3-6,8]$.

The NICHD MFMU network BEAM trial has been the subject of multiple secondary analyses addressing the relationship of magnesium sulfate dose, duration, or cord blood concentration with neonatal outcomes as it is the largest trial performed to investigate the effect of magnesium sulfate on neurodevelopmental outcomes and neonatal death [21-24]. Of note was a secondary analysis of the BEAM trial, investigating whether magnesium cord blood concentration and magnesium sulfate infusion at time of delivery are associated with cerebral palsy or death diagnosed by the age of 2 years [25]. Our 
analysis yielded comparable results to that study, demonstrating that there was no association between magnesium cord blood concentration or magnesium sulfate infusion with cerebral palsy or death at the time of delivery. However, the analysis by Palatnik el al [25]; included all non-anomalous singleton infants

whose mothers received magnesium sulfate, with only 509 (54\%) mothers received magnesium sulfate at the time of delivery. In our analysis, we included infants born to women who received magnesium sulfate only within 6 hours of delivery. This was essential because of magnesium rapid renal elimination from the maternal circulation. Also, we did not exclude twin gestations, as these are usually at higher risk of cerebral palsy compared with singletons. In our analysis, clustering was adjusted for multiple gestation. Additional analysis of the BEAM trial found that the duration of antenatal magnesium sulfate infusion is not associated with stillbirth or neonatal death or cerebral palsy [22]. That cord blood magnesium concentration was also not associated with delivery room resuscitation of neonates $[23,24]$.

Meta-analyses have found no change in rates of intraventricular hemorrhage, periventricular leukomalacia, Apgar scores less than 7 at 5 minutes, neonatal seizures, and chronic lung disease regardless of treatment (placebo vs. magnesium sulfate) [3, 8]. Our results found no association between cord blood magnesium concentration and intraventricular hemorrhage or periventricular leukomalacia. The sheer number of analyses addressing magnesium sulfate dosing, schedule, and effects, illuminates' 'clinicians' desires to find evidence-based guidance for antenatal magnesium sulfate use for fetal neuroprotection.

This is one of two studies only that investigate the relationship between cord blood magnesium concentration and child death/neurodevelopmental outcomes. However, compared to the previous secondary analysis [25] performed on the same data set, this study contains a larger number of neonates (668 compared with 596) as it includes patients who received magnesium sulfate within 6 hours of delivery and twin gestations. Also, we investigated additional outcomes such as abnormalities of neonatal head ultrasound findings (intraventricular hemorrhage and periventricular leukomalacia). Cord blood magnesium concentration is an accurate evaluation of the fetal magnesium concentration at the time of birth. Of note, the original study was powered to determine a difference in the outcomes for magnesium sulfate use versus control; it was not powered to detect a difference in the outcomes based on cord blood magnesium concentration. This study only included patients from the primary RCT who received magnesium sulfate, as we evaluated the association of cord blood magnesium concentrations and the outcomes previously specified. However, our study has several limitations, including being a retrospective analysis of a cohort from a prior conducted randomized trial, and a significant percentage of missing data, which might have limited our ability to detect any differences.

\section{Conclusions}

In conclusion, cord blood magnesium concentrations were not significantly associated with moderate to severe cerebral palsy or neonatal death, or other neurodevelopmental or sonographic outcomes. Further research should involve pharmacokinetic and pharmacodynamic modeling to evaluate the relationship between neonatal magnesium concentrations and child outcomes. This may be our best method to 
discover the ideal dose and schedule of magnesium sulfate for fetal neuroprotection, as it is unlikely that another larger trial will ever be conducted.

\section{Abbreviations}

ACOG - American College of Obstetricians and Gynecologists

ACTOMgSO4 - Australasian Collaborative Trial of Magnesium Sulphate

BEAM - Beneficial Effects of Antenatal Magnesium

$\mathrm{CP}$ - cerebral palsy

NICHD-MFMU - National Institutes of Child Health and Human Development Maternal-Fetal Medicine Units

RCT - randomized controlled trials

SMFM - Society for Maternal-Fetal Medicine

\section{Declarations}

Ethics approval and consent to participate: This secondary analysis was exempt from review by the University of Texas Medical Branch Institutional Review Board, as it met the criteria of research involving the study of existing data.

Consent for publication: not applicable

Availability of data and materials: This is a secondary analysis of data from the Eunice Kennedy Shriver National Institutes of Child Health and Human Development Maternal-Fetal Medicine Units Network multicenter randomized trial (Beneficial Effects of Antenatal Magnesium [BEAM])

Competing interests: We declare that Dr. Catherine Sherwin is a Section Editor for Clinical Pharmacology for the BMC Pharmacology and Toxicology journal. The authors declare they have no other competing interests.

Funding: There were no sources of support for this study.

Authors' contributions: CMS and MC developed the idea for this study, CLD and XL extracted data for this study. CLD, XL, AB, MC, and CMS developed the study design, CLD, XL, $A B$, completed the data analysis, and CLD, and XL wrote the initial manuscript. CMS and MC provided clinical consultation on the study work. All authors reviewed the manuscript, provided critical feedback, and approved its journal submission. 
Acknowledgments: The authors would like to thank the Eunice Kennedy Shriver National Institutes of Child Health and Human Development and the Maternal-Fetal Medicine Units Network for access to the data from the Beneficial Effects of Antenatal Magnesium [BEAM] and to thank Luzviminda (Luz) Sinha, Medical and Research Librarian at Dayton Children's Hospital, for her edits and review.

Authors' information: C Luke Dixon and Maged Costantine, Department of Obstetrics and Gynecology, The University of Texas Medical Branch at Galveston, TX; Xiaoxi Liu and Alfred Balch, Division of Clinical Pharmacology, Department of Pediatrics, School of Medicine, University of Utah, Salt Lake City, UT.

Catherine M. Sherwin, Department of Pharmacotherapy, College of Pharmacy, University of Utah, Salt Lake City, UT; Department of Pediatrics, Wright State University Boonshoft School of Medicine/Dayton 'Children's Hospital, Dayton, $\mathrm{OH}$

\section{References}

1. Wimalasundera N, Stevenson VL. Cerebral palsy. Pract Neurol. 2016;16(3):184-194

2. Maenner MJ, Blumberg SJ, Kogan MD, Christensen D, Yeargin-Allsopp M, Schieve LA. Prevalence of cerebral palsy and intellectual disability among children identified in two U.S. National Surveys, 2011-2013. Ann Epidemiol. 2016;26(3):222-226

3. Doyle LW, Crowther CA, Middleton P, Marret S, Rouse D. Magnesium sulphate for women at risk of preterm birth for neuroprotection of the fetus. Cochrane Database Syst Rev. 2009(1):CD004661

4. Crowther CA, Hiller JE, Doyle LW, Haslam RR, Group ACToMSASC. Effect of magnesium sulfate given for neuroprotection before preterm birth: a randomized controlled trial. JAMA. 2003;290(20):26692676

5. Marret S, Marpeau L, Zupan-Simunek V, et al. Magnesium sulphate given before very-preterm birth to protect infant brain: the randomised controlled PREMAG trial*. BJOG. 2007;114(3):310-318

6. Rouse DJ, Hirtz DG, Thom E, et al. A randomized, controlled trial of magnesium sulfate for the prevention of cerebral palsy. N Engl J Med. 2008;359(9):895-905

7. Costantine MM, Weiner SJ, Network EKSNIoCHaHDM-FMU. Effects of antenatal exposure to magnesium sulfate on neuroprotection and mortality in preterm infants: a meta-analysis. Obstet Gynecol. 2009;114(2 Pt 1):354-364

8. Conde-Agudelo A, Romero R. Antenatal magnesium sulfate for the prevention of cerebral palsy in preterm infants less than 34 weeks' gestation: a systematic review and meta-analysis. Am J Obstet Gynecol. 2009;200(6):595-609

9. Committee Opinion No 652: Magnesium Sulfate Use in Obstetrics. Obstet Gynecol. 2016;127(1):e5253

10. Rouse DJ. Magnesium sulfate for fetal neuroprotection. Am J Obstet Gynecol. 2011;205(4):296-297

11. Sibai BM. Magnesium sulfate for neuroprotection in patients at risk for early preterm delivery: not yet. Am J Obstet Gynecol. 2011;205(4):296-297 
12. Cahill AG, Stout MJ, Caughey AB. Intrapartum magnesium for prevention of cerebral palsy: continuing controversy? Curr Opin Obstet Gynecol. 2010;22(2):122-127

13. Jacquemyn $Y$, Zecic $A$, Van Laere $D$, Roelens $K$. The use of intravenous magnesium in nonpreeclamptic pregnant women: fetal/neonatal neuroprotection. Arch Gynecol Obstet. 2015;291(5):969-975

14. Huusom LD, Secher NJ, Pryds O, Whitfield K, Gluud C, Brok J. Antenatal magnesium sulphate may prevent cerebral palsy in preterm infants-but are we convinced? Evaluation of an apparently conclusive meta-analysis with trial sequential analysis. BJOG. 2011;118(1):1-5

15. Doyle LW, Anderson PJ, Haslam R, Lee KJ, Crowther C, Group ACToMSAS. School-age outcomes of very preterm infants after antenatal treatment with magnesium sulfate vs placebo. JAMA. 2014;312(11):1105-1113

16. Rigo J, Pieltain C, Christmann V, et al. Serum Magnesium Levels in Preterm Infants Are Higher Than Adult Levels: A Systematic Literature Review and Meta-Analysis. Nutrients. 2017;9(10):1125

17. Rouse DJ. Magnesium sulfate for the prevention of cerebral palsy. Am J Obstet Gynecol. 2009;200(6):610-612

18. Brookfield KF, Su F, Elkomy MH, Drover DR, Lyell DJ, Carvalho B. Pharmacokinetics and placental transfer of magnesium sulfate in pregnant women. Am J Obstet Gynecol. 2016;214(6):737.e731-739

19. Brookfield KF, Elkomy M, Su F, Drover DR, Carvalho B. Optimization of Maternal Magnesium Sulfate Administration for Fetal Neuroprotection: Application of a Prospectively Constructed Pharmacokinetic Model to the BEAM Cohort. J Clin Pharmacol. 2017

20. Mittendorf R, Dambrosia J, Pryde PG, et al. Association between the use of antenatal magnesium sulfate in preterm labor and adverse health outcomes in infants. Am J Obstet Gynecol. 2002;186(6):1111-1118

21. McPherson J, Smiley S, Stamilio D. Maternal obesity and neuroprotective magnesium sulfate. Am J Obstet Gynecol. 2015;213(4):582.e581-586

22. McPherson JA, Rouse DJ, Grobman WA, Palatnik A, Stamilio DM. Association of duration of neuroprotective magnesium sulfate infusion with neonatal and maternal outcomes. Obstet Gynecol. 2014;124(4):749-755

23. Johnson LH, Mapp DC, Rouse DJ, et al. Association of cord blood magnesium concentration and neonatal resuscitation. J Pediatr. 2012;160(4):573-577.e571

24. Drassinower D, Friedman AM, Levin H, Običan SG, Gyamfi-Bannerman C. Does magnesium exposure affect neonatal resuscitation? Am J Obstet Gynecol. 2015;213(3):424.e421-425

25. Palatnik, A, Rouse DJ, Stamilio DM, et al. Associations between cerebral palsy or death and umbilical cord blood magnesium concentrations. Am J Perintaol 2015; 32:1263-7 